\title{
Using Principal Component Analysis and Cluster Analysis to determine agricultural capacities of Algeria
}

\author{
Houssame Eddine BALOULI \\ PhD student at ENSSEA \\ Balouli.houssame.edddine@gmail.com \\ Dr. Lazhar CHINE \\ Boumerdes University \\ I.chnie@univ-boumerdes.dz
}

\begin{abstract}
This work aims to apply principal components analysis and cluster analysis to determine the Agricultural capacities of Algeria. We find four agricultural areas. These areas characterized by the production of citrus fruits, potatoes and potatoes, cereals and finally Livestock.
\end{abstract}

Key words

Principal Components Analysis. Cluster Analysis. Agriculture. Algeria

الملخص

يهدف هذا العمل إلى تطبيق التحليل عن طريق المركبات الأساسية والتحليل العنقودي من أجل تحديد أهم القدرات الفلاحية للجزائر. بعد تحليل النتائج وجدنا أن الجزائر تتميز بأربعة مناطق فلاحية. تتميز هذه المناطق الفلاحية بإنتاج الحمضيات والفواكه؛ البطاطا والطماطم؛ الخضر والحبوب؛ وأخيرا تربية المواشي. الكثمات المفتاحية

التحليل عن طريق المركبات الأساسية. التحليل العنقودي. الفلاحة. الجزائر 


\section{Introduction}

Principal components analysis (PCA) is a factor method, which initiated by Pearson in 1901, and developed by Hotelling in 1933 (Rotaru et al, 2012). It is a useful statistical technique for finding patterns in data of high dimension. It has found application in several fields such as agriculture, finance, economics etc. PCA is a procedure by which numbers of correlated variables are transformed into a smaller number of uncorrelated variables. It can be used to compress a high dimensional dataset into a lower dimensional dataset. PCA is based on the minimization of the mean square error in data compression, finding mutually orthogonal directions in the data having maximal variances and decorrelation of the data using orthogonal transformation. These uncorrelated variables are called Principal Components (Jyotirmoy Sarkara et al, 2014).

Cluster analysis is a collection of relatively simple descriptive statistical techniques with potential value in several fields. There are many methods of cluster analysis. One of them is the classification, which is an essential means of organizing information (Jane Clatworthy et al, 2005).

\section{Data sets}

We try to apply Principal Component Analysis (PCA) and Cluster Analysis (CA) on a data table of 48 individuals and 19 variables using statistical programing language $\mathrm{R}$. The 48 individuals are the 48 states of Algeria. The 19 variable are a statistics about Plant wealth, Livestock and some geographical characteristics such as the geographical location, height and number of dams. The database is loaded from National Office of Statistics.

\section{Finding and Discussion}

\subsection{The preparation of the database}

Read the database using read.csv2:

\section{setwd("C:/Users/houssem/Downloads/Colloques/Workshop-ENSSEA2017")}

Agr_cap_Alg <- read.csv2("Agr_cap_Alg.csv", header = TRUE, sep = ";", check.names = FALSE, row.names $=1$ )

- setwd: setting working directory. Choosing the location of the file as a working directory.

- Agr_cap_Alg: file's name;

- read.csv2: The command used to read the file. The "csv" means comma-separated values.

- header = TRUE: the first row represents the names of variables.

- $\quad$ sep = ";": means that ";" separates the information.

- $\quad$ check.names = FALSE: accept the names of variables as they.

- row.names $=1$ : the first row represents the names of the individuals.

View the database using view:

View(Agr_cap_Alg)

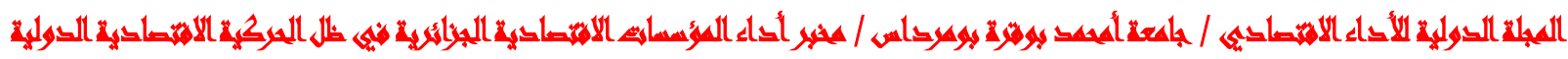
العقى الاول - ميوان 2018 
Summarize and View the structure and of the database using summary

\begin{tabular}{|c|c|c|c|c|c|c|}
\hline \multicolumn{2}{|r|}{ Cows } & \multicolumn{2}{|c|}{ Bulls } & \multicolumn{2}{|c|}{ Sheep } & Caprine \\
\hline Min. & $: \quad 58$ & Min. & : $\quad 70$ & Min. & : 22244 & Min. : 3485 \\
\hline 1st Qu. & : 7806 & 1st Qu. & : 14590 & 1st Qu.: & : 160317 & 1st Qu.: 32867 \\
\hline Median & :18020 & Median & : 33000 & Median : & : 385750 & Median : 65380 \\
\hline Mean & $: 21926$ & Mean & : 41510 & Mean & : 553604 & Mean : :102306 \\
\hline 3rd Qu. & $: 26450$ & 3rd Qu. & : 51526 & 3rd Qu.: & : 653099 & 3rd Qu.: 126566 \\
\hline Max. & : 79941 & Max. & :132270 & Max. : & : 3113500 & Max. $: 526000$ \\
\hline NA's & $: 2$ & NA's & $: 2$ & & & \\
\hline
\end{tabular}

The summary command allows us to summarize the data by calculating indicators of descriptive statistics. These indicators are the minimum, the first quartile, the median, the mean, the third quartile, the maximum and the number of missing values (NA).

\subsection{Manage missing data}

We have several methods to replace the missing values:

- Eliminates the variables and the individuals that contain the missing values

- by the mean

- Using ("missMDA") function. We call this method Iterated Principal Component Analysis.

Install and load the package

install.packages("missMDA")

library(missMDA)

New_Agr_cap_Alg $<-$ imputePCA(Agr_cap_Alg[, 1:18], scale = TRUE)

- New_Agr_cap_Alg: The new file that contain all the values.

- imputePCA: The command used to replace the missing values.

Save the new database in a csv file using write.csv2

write.csv2(New_Agr_cap_Alg, file = "New_Agr_cap_Alg.csv")

\subsection{Execute Principal Component Analysis using FactoMiner}

Install and load the package and execute the PCA analysis

install.packages("FactoMiner")

library(FactoMineR)

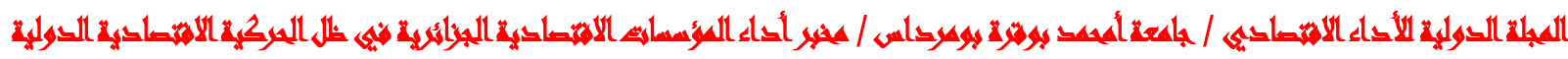
العتى الاول - ميوان 2018 
Princ_Comp_Ana $<-$ PCA(New_Agr_cap_Alg, quali.sup $=19$, quanti.sup = 16)

- Princ_Comp_Ana: the object that contain the results of PCA.

- PCA: the command used to execute the PCA analysis.

- quali.sup $=19$ : The $19^{\text {th }}$ variable is a supplementary qualitative variable.

- quanti.sup $=16$ : the $16^{\text {th }}$ variable is supplementary quantitative variable.

The main results of PCA are:

Figure 01: Individuals map

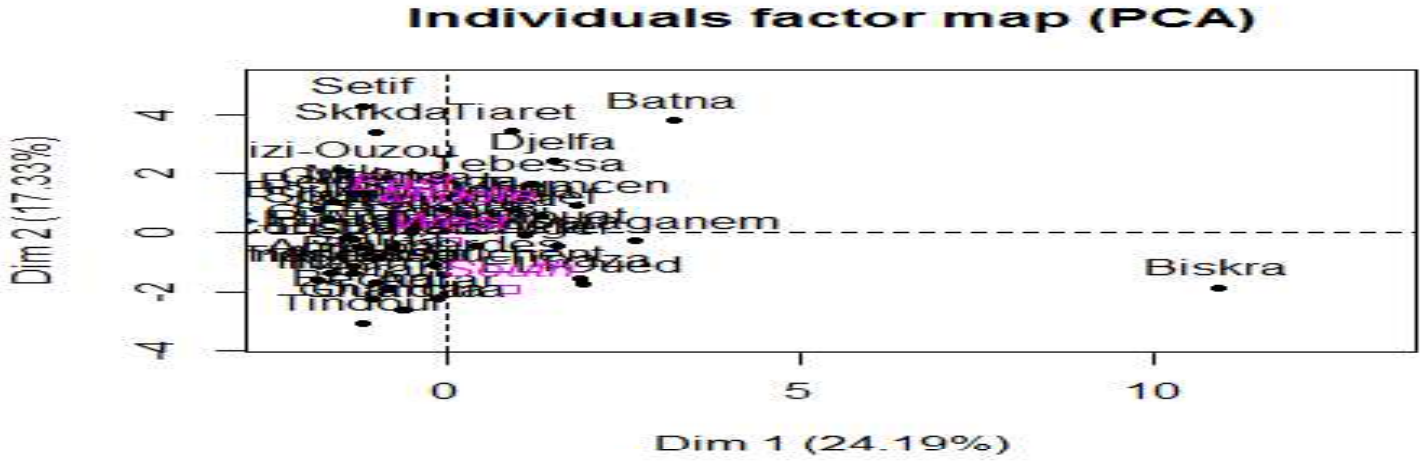

Source: R outputs

Figure 02: variables map

Variables factor map (PCA)

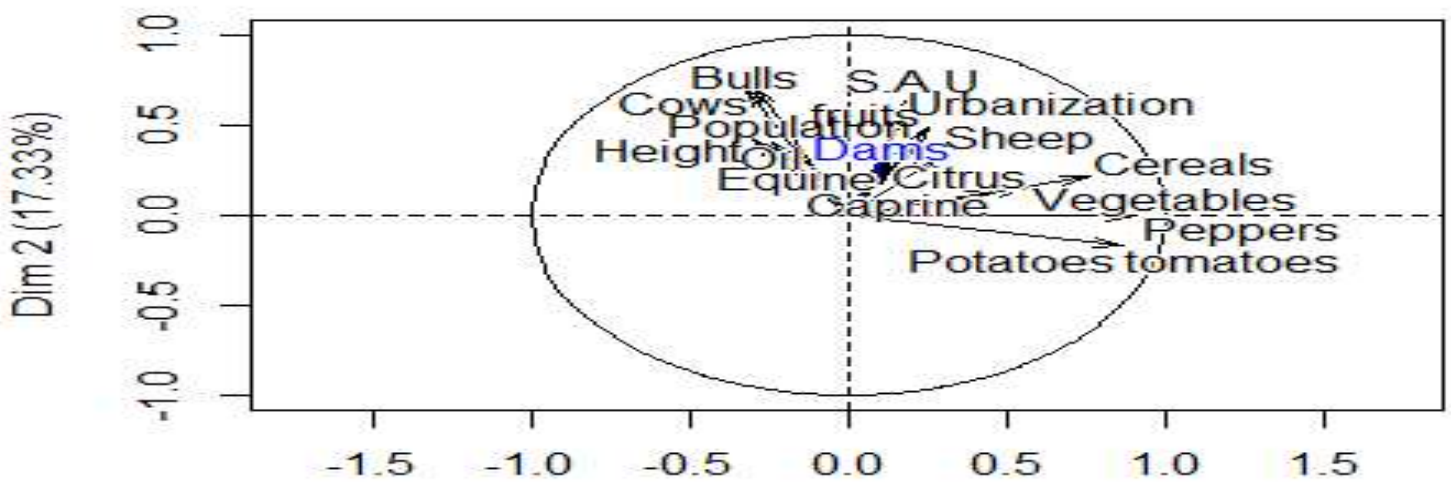

Dim $1(24.19 \%)$

Source: R outputs

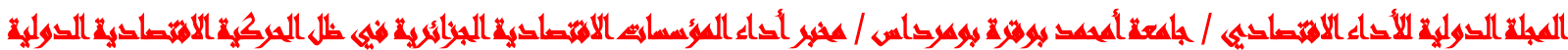
2018 العقى الاول - 2015 
We find that the graphs are not readable and ambiguous regarding to the position of individuals and variables. We cannot interpret these maps. We will use another package to improve the results of PCA.

A summary of the main results can be obtained using summary function. The Eigenvalues, the coordinates (Dim), the quality of representation $(\cos 2)$ and the contribution (ctr) of each variable and individual on each axe.

summary(Princ_Comp_Ana)

Eigenvalues

$\begin{array}{lrrrrrr} & \text { Dim.1 } & \text { Dim.2 } & \text { Dim.3 } & \text { Dim.4 } & \text { Dim.5 } & \text { Dim.6 } \\ \text { Variance } & 4.112 & 2.945 & 2.399 & 1.701 & 1.066 & 0.940 \\ \text { \% of var. } & 24.191 & 17.326 & 14.110 & 10.003 & 6.271 & 5.532 \\ \text { Cumulative \% of var. } & 24.191 & 41.517 & 55.627 & 65.630 & 71.901 & 77.433\end{array}$

Individuals (the 10 first)

\begin{tabular}{|c|c|c|c|c|c|c|c|}
\hline & Dist & Dim.1 & ctr & $\cos 2$ & Dim. 2 & ctr & $\cos 2$ \\
\hline Adrar & 2.715 & -0.153 & 0.012 & 0.003 & -2.253 & 3.592 & 0.689 \\
\hline Chlef & 3.186 & 1.302 & 0.859 & 0.167 & 0.569 & 0.229 & 0.032 \\
\hline Laghouat & 4.115 & 1.091 & 0.603 & 0.070 & -0.111 & 0.009 & 0.001 \\
\hline O.E.Bouaghi & 2.080 & -0.497 & 0.125 & 0.057 & 0.023 & 0.000 & 0.000 \\
\hline Batna & 6.283 & 3.210 & 5.219 & 0.261 & 3.792 & 10.171 & 0.364 \\
\hline Bejaia & 5.552 & -1.591 & 1.282 & 0.082 & 1.073 & 0.814 & 0.037 \\
\hline Biskra & 11.692 & 10.893 & 60.106 & 0.868 & -1.868 & 2.468 & 0.026 \\
\hline Bechar & 2.940 & -1.045 & 0.553 & 0.126 & -2.235 & 3.534 & 0.578 \\
\hline Blida & 7.517 & 0.205 & 0.021 & 0.001 & 1.095 & 0.848 & 0.021 \\
\hline Bouira & 2.857 & -1.842 & 1.719 & 0.416 & 0.784 & 0.435 & 0.075 \\
\hline
\end{tabular}

Variables (the 10 first)

\begin{tabular}{l|rrr|rrr|rr} 
& Dim.1 & ctr & cos2 & Dim.2 & ctr & $\cos 2$ & Dim.3 & ctr \\
Cows & -0.292 & 2.072 & 0.085 & 0.711 & 17.149 & 0.505 & 0.312 & 4.067 \\
Bulls & -0.319 & 2.479 & 0.102 & 0.697 & 16.508 & 0.486 & 0.335 & 4.665 \\
Sheep & 0.290 & 2.050 & 0.084 & 0.345 & 4.043 & 0.119 & -0.746 & 23.210 \\
Caprine & 0.467 & 5.298 & 0.218 & 0.135 & 0.616 & 0.018 & -0.525 & 11.509 \\
Equine & 0.122 & 0.363 & 0.015 & 0.277 & 2.602 & 0.077 & -0.633 & 16.728
\end{tabular}

\subsection{Principal Component Analysis using factoextra}

install.packages("factoextra")

library("factoextra")

Princ_Comp_Ana $<-$ princomp(New_Agr_cap_Alg)

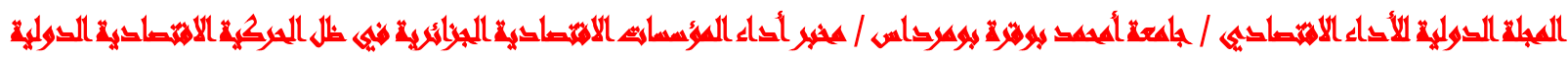
العتى الأول - يجان 2018 
- Princ_Comp_Ana: the object that contain the results of PCA using factoextra

- Princomp: the command used to execute PCA.

Visualize eigenvalues (scree plot). Show the percentage of variances explained by each principal component:

fviz_eig(Princ_Comp_Ana)

Figure 03: eigenvalues

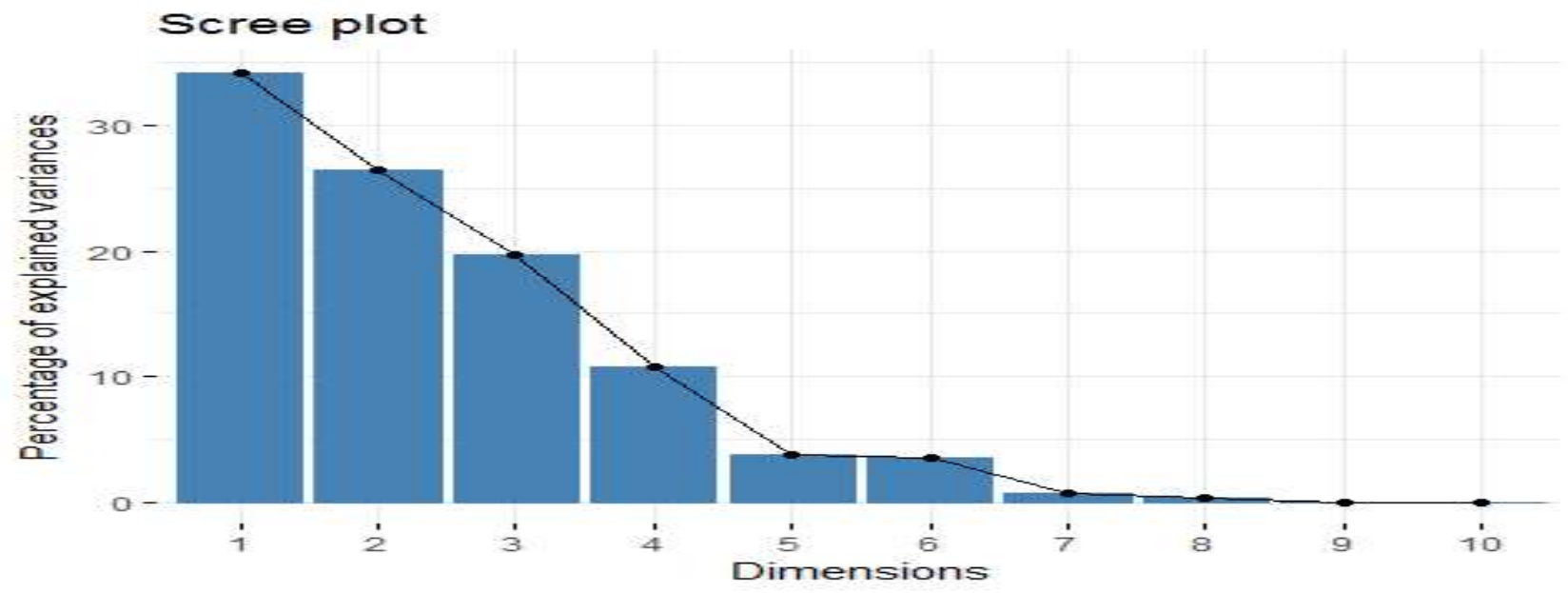

Source: R outputs

Graph of individuals using fviz_pea_ind function for the first and the second axes

fviz_pca_ind(Princ_Comp_Ana, axes $=c(1,2)$, select.ind $=$ list $(\cos 2=20)$, col.ind = "cos2", \# Color by the quality of representation gradient.cols = c("\#00AFBB", "\#E7B800", "\#FC4E07"), repel = TRUE \# Avoid text overlapping

Figure 04: Individuals map

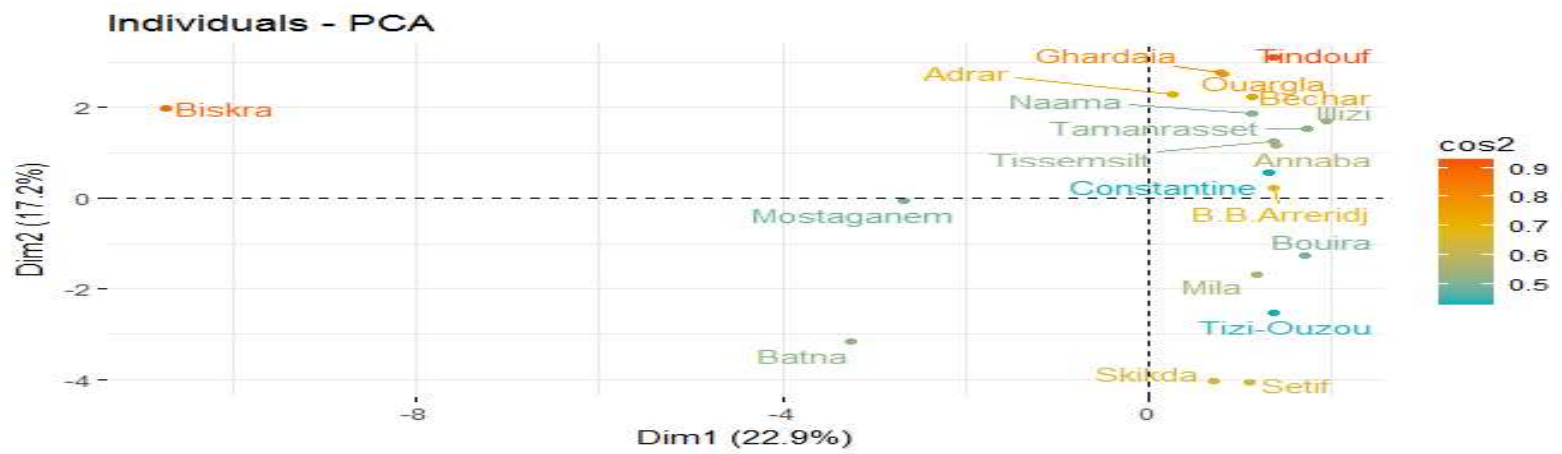

Source: R outputs

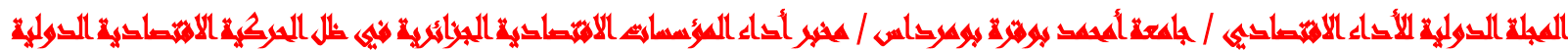
2018 العقى الاول - 24 
According to this map, the individuals who have a better representation on the first factorial plane are Tindouf, Biskra, Ghardaia, Bechar and Ouargla.

Graph of variables using fviz_pca_var function for the first factorial plan

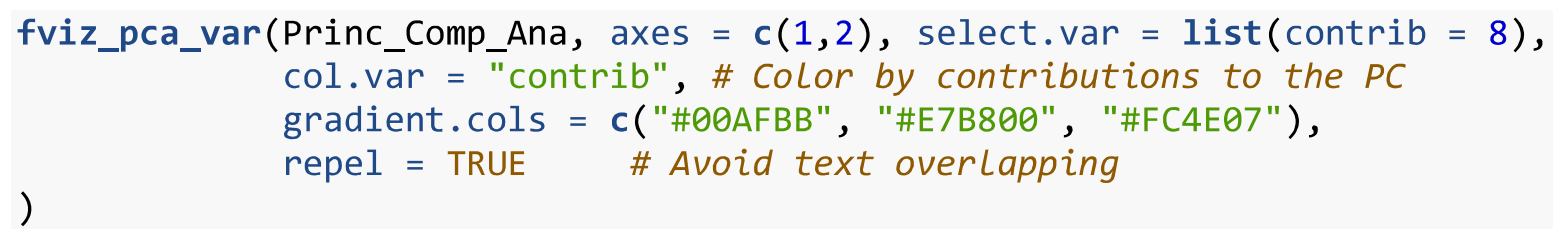

\section{Figure 05: variables map}

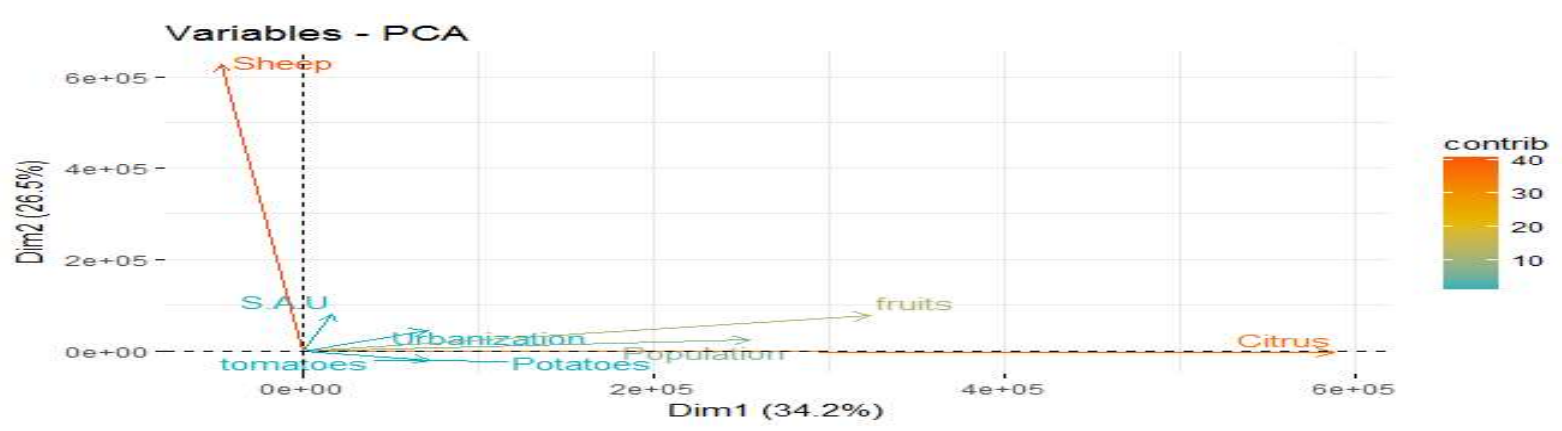

Source: R outputs

The variables that contribute the most in the construction of the first two axes are Citrus and Sheep. Biplot of individuals and variables

fviz_pca_biplot(Princ_Comp_Ana, axes $=\mathbf{c}(1,2)$, repel $=$ TRUE, select.ind $=$ list $(\cos 2=15)$, select.var $=\operatorname{list}(\cos 2=8)$,

col.var = "\#2E9FDF", \# Variables color

col.ind $=" \# 696969 "$ \# Individuals color

\section{Figure 06: Biplot of individuals and variables}

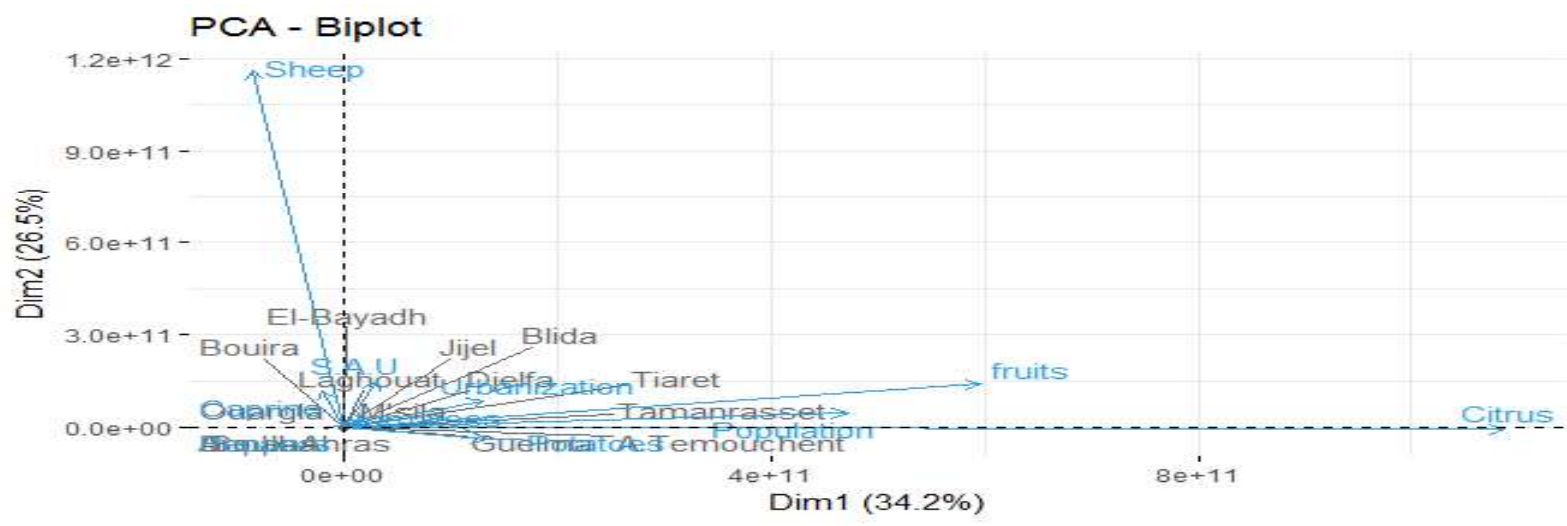

Source: R outputs

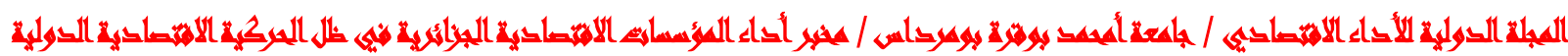
العقى الاول - يوان 2018 


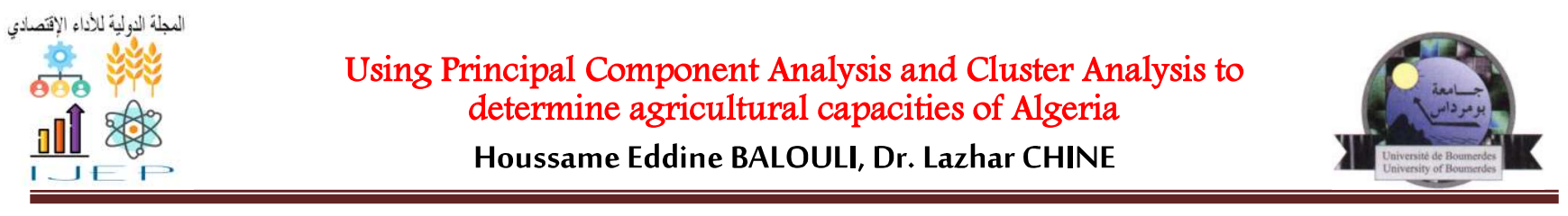

We repeat the same analysis with the third and the fourth axes $c(1,3), c(1,4), c(2,3)$ and $c(3,4)$.

The individuals who have a better representation on the other factorial planes are Skikda and Adrar, Biskra, Blida, Djelfa, M'sila, Tiaret, Relizane and El-Oued. The variables that contribute the most in the construction of the other factorial planes are Potatoes, tomatoes and fruits.

\subsection{The matrix correlation}

install.packages("corrplot")

library (corrplot)

Cor_matrix <- cor(New_Agr_cap_Alg)

corrplot(Cor_matrix, order $=$ "AOE", method = "color", addCoef.col = "gray", is.corr = FALSE )

corrplot.mixed(Cor_matrix)

Figure 07: Correlation matrix

\begin{tabular}{|c|c|c|c|c|c|c|c|c|c|c|c|c|c|c|c|c|c|}
\hline ows & 。 & 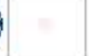 & $\theta$ & & 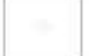 & 0 & 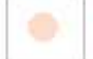 & $\theta$ & 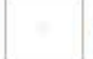 & 0 & & $\theta$ & 0 & 8 & (1) & $\theta$ & 0 \\
\hline 0.98 & Bulls & a & 당 & 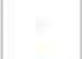 & & 8 & 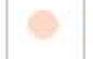 & (1) & a & 0 & & 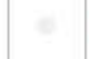 & 0 & O & 8 & & P \\
\hline & & sheep & 0 & 0 & $\theta$ & & & (1) & (1) & 0 & a & 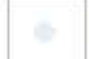 & $\theta$ & 0 & $\theta$ & & (1) \\
\hline 0.15 & -0.1 & 0.61 & aprin & e & 8 & $\odot$ & e & 0 & 0 & $\theta$ & $\theta$ & 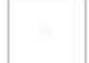 & $\theta$ & O & $\circ$ & 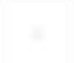 & 6 \\
\hline & & 0.51 & 0.21 & quine & & 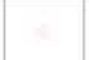 & 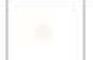 & & . & 0 & . & & 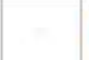 & 8 & 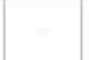 & & $\theta$ \\
\hline & & 0.1 & $|0.19|$ & & getab & oles & 0 & & 0 & $\theta$ & 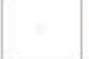 & $\theta$ & 8 & 0 & 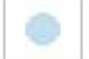 & 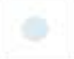 & $\theta$ \\
\hline 0.18 & -0.2 & & 0.2 & & $0.42 p$ & tatoe & & & & $=$ & 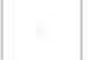 & An & 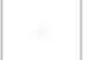 & 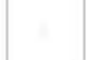 & 6 & 0 & 0 \\
\hline 0.18 & -0.2 & & 0.2 & & 0.42 & 1 & natoe & & 0 & a & 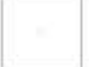 & $=$ & 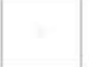 & 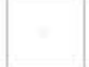 & 0 & 0 & 0 \\
\hline-0.19 & -0.2 & 0.22 & 0.33 & & 0.48 & 0.85 & 0.85 & eppe & 15 & $\theta$ & a & $\theta$ & 8 & $\theta$ & $\odot$ & 9 & $\theta$ \\
\hline & & 0.23 & 0.43 & & 0.34 & 0.56 & 0.56 & 0.72 & ereal & Is & 6 & 0 & (1) & 0 & & & \\
\hline 0.41 & 0.45 & 0.18 & 0.14 & 0.18 & 0.09 & 0.0 & t & 0.11 & 0.11 & Oil & 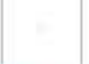 & $\theta$ & (1) & 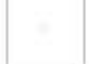 & 0 & 0 & $\theta$ \\
\hline & & & 0.13 & & & & & & 0.13 & & Citrus & 50 & (1) & $\theta$ & & 0 & 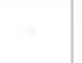 \\
\hline 0.11 & 209 & 0.41 & & & 0.14 & 100 & t & 0.18 & 0.33 & 0.15 & 0.7 & fruits & 0 & P & (1) & 0 & z \\
\hline 0.21 & 0.22 & 0.12 & 0.16 & & 0.19 & & & 0.13 & 0.2 & 0.26 & 0.2 & 0.22 & anizat & tion & 0 & 0 & 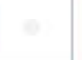 \\
\hline 0.25 & 0.23 & 0.52 & 0.22 & 0.55 & 0.24 & & & 0.17 & 0.24 & & & 0.21 & $0.37=$ & SA.U & 10 & 8 & ○ \\
\hline 0.29 & 0.29 & & 0.16 & & 0.24 & $0,16)$ & 0.16 & 0.15 & & 0,19 & & 0.28 & 0.31 & 0.21 & Dams & & \\
\hline 0.17 & 0.18 & & & & 0.15 & 0.19 & 0.19 & & 0.17 & 0.18 & 0.31 & 0.35 & 0.28 & 0.19 & & pulati & on \\
\hline 0.25 & 0.24 & 0.22 & 0.07 & 0.1 & $-0,18$ & -0.38 & $3 \cdot 0.38$ & 0.18 & & 0.14 & & & 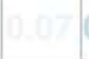 & 0.29 & & -0.12 & teight \\
\hline
\end{tabular}

Source: R outputs

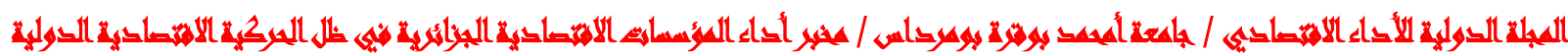
العقى الاول - يوان 2018 
The most important correlation is between cows and bulls (ctr $=0.98)$, potatoes and peppers $(\mathrm{ctr}=0.85)$, tomatoes and peppers $(\mathrm{ctr}=0.85)$ and between citrus and fruits $(\mathrm{ctr}=0.7)$.

\subsection{Cluster Analysis}

library(dendextend)

library(factoextra)

library(igraph)

dd <- dist(scale(New_Agr_cap_Alg), method = "euclidean")

hc $<-$ hclust (dd, method = "ward.D2")

We can use the arguments main, sub, xlab, ylab to change plot titles as follow

fviz_dend(hc, cex $=0.5$, main = "Dendrogram - ward.D2", xlab = "Objects", ylab = "Distance", sub = "'")

fviz_dend(hc, $k=4$, \# Cut in four groups

cex $=0.5$, \# Label size

k_colors = c("\#2E9FDF", "\#00AFBB", "\#E7B800", "\#FC4E07"),

color_labels_by_k = TRUE, \# color Labels by groups

ggtheme = theme_gray () \# Change theme

Additionally, we can plot a circular dendrogram using the option type = "circular".

fviz_dend(hc, cex $=0.5, \mathrm{k}=4$,

$\mathrm{k}$ colors $=$ "jco", type $=$ "circular")

Figure 08: Cluster Dendrogram

Cluster Dendrogram

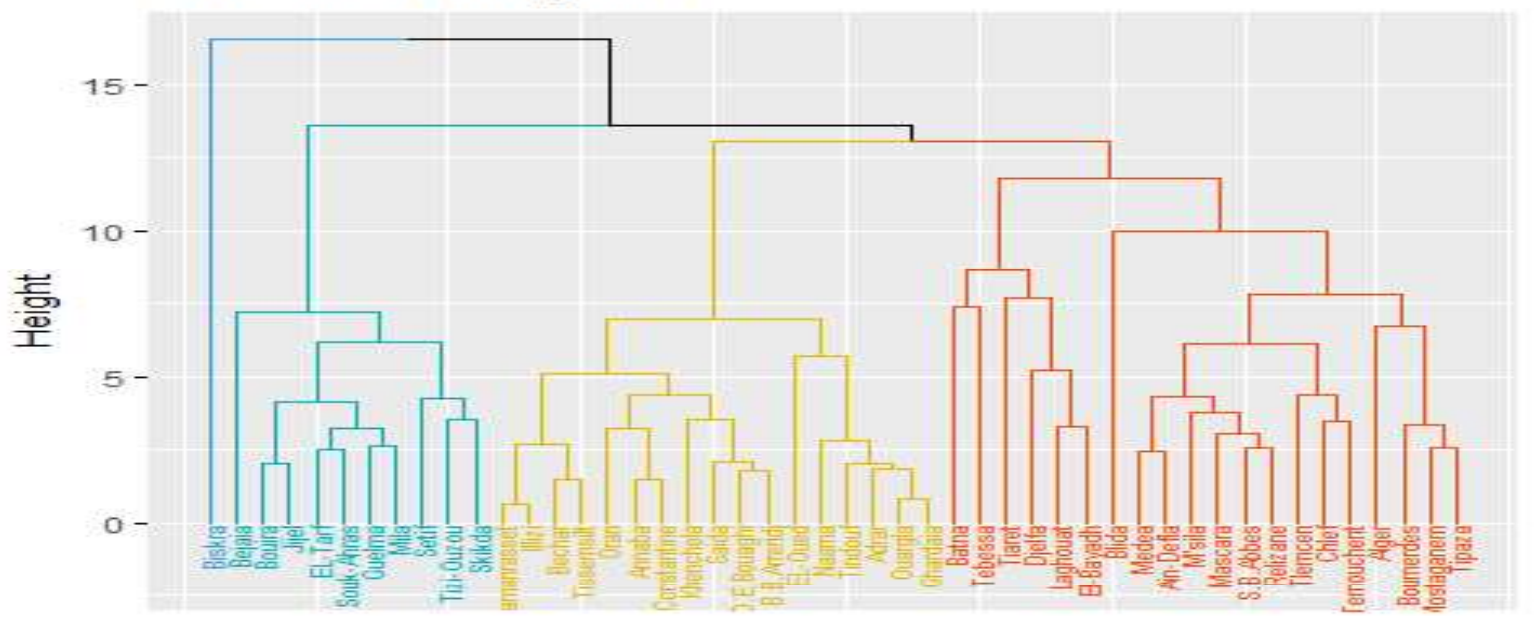

Source: R outputs

Additionally, we can plot a circular dendrogram using the option type $=$ "circular".

fviz_dend(hc, cex $=0.5, \mathrm{k}=4$,

k_colors $=$ "jco", type $=$ "circular")

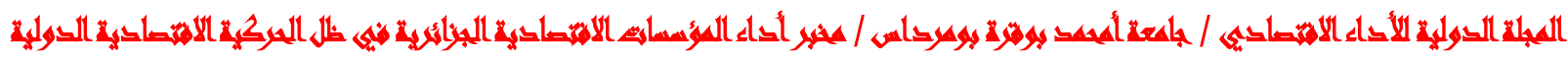
العقى الاول - ميوان 2018 


\section{Figure 10: circular dendrogram}

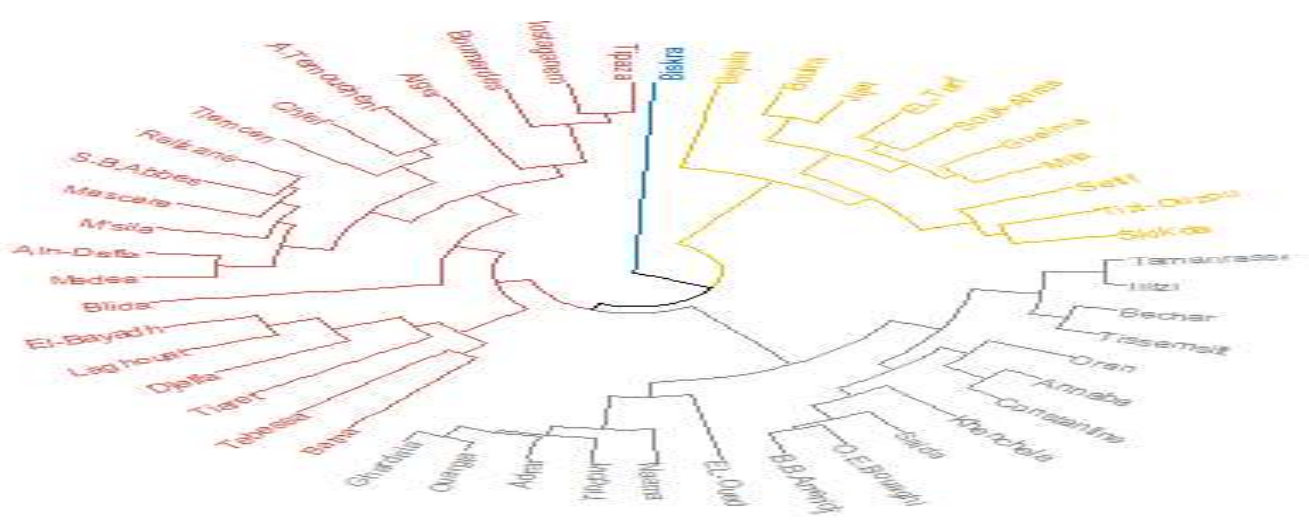

Source: R outputs

To plot a phylogenic-like tree, we use type $=$ "phylogenic" and repel = TRUE (to avoid labels overplotting). This functionality requires the $\mathrm{R}$ package igraph. Make sure that it is installed before typing the following $\mathrm{R}$ code.

fviz_dend(hc, $\mathrm{k}=4$, $\mathrm{k} \_$colors $=$"jco",

type $=$ "phylogenic", repel $=$ TRUE)

Figure 11: tree dendrogram

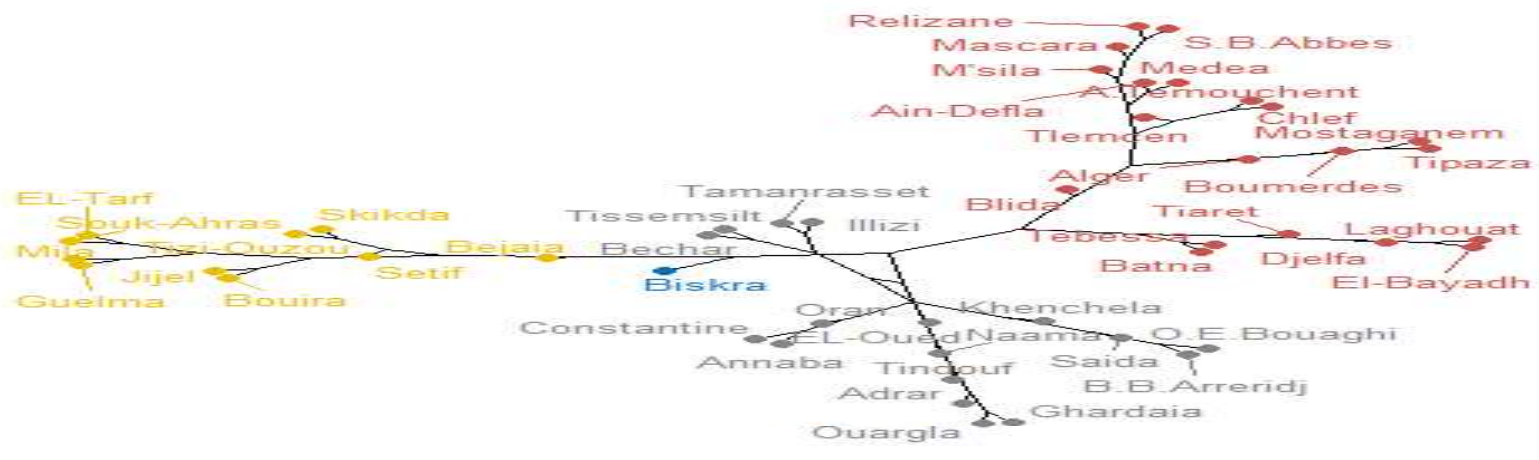

Source: R outputs

According to the results of the cluster analysis, we have four groups (clusters). The first cluster is Biskra. It is the most important agricultural state in Algeria. It is characterized by the production of potatoes and tomatoes in addition to the production of dates. The second cluster is Blida, Tipaza, Alger, Ain-Defla Mascara and Relizane, which characterized by the production of citrus and fruits. The third cluster is Djelfa, Laghouat and M'sila, which characterized by Livestock. The fourth is Khenchela, Batna, Constantine and Annaba, which characterized by the production of cereals.

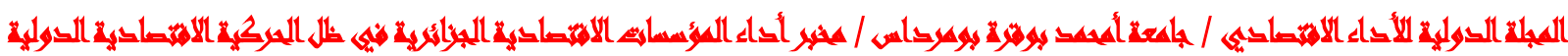

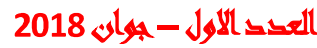




\section{Conclusion}

Since its inception, R software has become an essential tool for statistics and data visualization in both the academic and corporate worlds. This exceptional development is explained by its three main qualities: it is free, very complete and in constant expansion.

Principal Component Analysis (PCA) is a dimension-reduction tool that can be used to reduce a large set of variables to a small set that still contains most of the information in the large set.

The objective of cluster analysis is to assign individulas (observations) to homogenus clusters (groups) so that observations within each group are similar to one another with respect to variables or attributes of interest.

Algeria can be divided into four agricultural regions (agricultural poles) or agricultural areas. These areas characterized by the production of citrus fruits; potatoes and potatoes; cereals; and finally Livestock. 


\section{Bibliography}

Isoz, V., Kolani, D. (2017). La Bible R en images (et en couleurs...). oUUID.

Kassambara, A. (2017). Practical Guide to Cluster Analysis in R: Unsupervised Machine Learning (Vol. 1). STHDA.

ROTARU, A. S., POP, I. D., VATCĂ, A., \& CIOBAN, A. (2012). Usefulness of Principal Components Analysis in Agriculture. Bulletin of the University of Agricultural Sciences \& Veterinary Medicine Cluj-Napoca. Horticulture, 69(2).

Sarkar, J., Saha, S., \& Agrawal, S. (2014). An Efficient Use of Principal Component Analysis in Workload Characterization-A Study. AASRI Procedia, 8, 68-74.

Lê, S., Josse, J. \& Husson, F. (2008). FactoMineR: An R Package for Multivariate Analysis. Journal of Statistical Software. 25(1). pp. 1-18.

Wei, T., Simko, V., Levy, M., Xie, Y., Jin, Y., \& Zemla, J. (2017). Package 'corrplot'. Statistician, 56, 316-324.

Kassambara, A. (2015). Factoextra: Visualization of the outputs of a multivariate analysis. $R$ package version, $1(1)$.

Csardi, G., \& Nepusz, T. (2006). The igraph software package for complex network research. InterJournal, Complex Systems, 1695(5), 1-9.

Josse, J., \& Husson, F. (2016). missMDA: a package for handling missing values in multivariate data analysis. Journal of Statistical Software, 70(1), 1-31.

Galili, T. (2015). dendextend: an R package for visualizing, adjusting and comparing trees of hierarchical clustering. Bioinformatics, 31(22), 3718-3720.

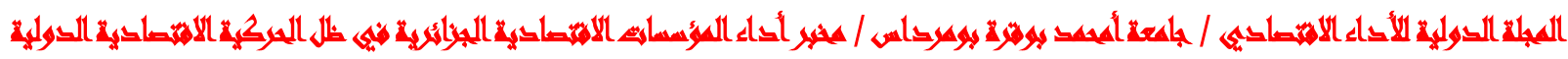
العتى الاول - ميوان 2018 